\title{
Schriftbildlichkeit: Etymographie und Ikonographie
}

Wenn man unter Etymo-logie die Erschließung eines Begriffs aus den innersprachlichen Beziehungen des Wortes versteht, das ihn bezeichnet und dieses Wort von daher als motiviert und nicht etwa arbiträr auffasst, dann wäre unter Etymo-graphie die ErschlieBung eines Begriffs aus seinem in welchem Sinne auch immer motivierten Schriftbild bzw. seiner Schriftbildlichkeit zu verstehen. Dieses Verfahren vermutet im Kontext einer Alphabetschrift, wie wir sie verwenden, einigermaßen abwegig an, obwohl es auch hier durchaus Ansätze in dieser Richtung gibt. Im Kontext einer Bilderschrift aber wie etwa der ägyptischen Hieroglyphen nimmt dieses Verfahren einen ganz zentralen Platz ein. In meinem Beitrag zu dem von Aleida Assmann und mir herausgegebenen Band über Hieroglyphen habe ich dieses Verfahren in gebührender Ausführlichkeit darzustellen versucht. ${ }^{1}$ Wenn ich hier nun trotzdem noch einmal darauf zurückkomme, dann einfach deshalb, weil ich den Eindruck habe, dass es im Kontext der Schriftbildlichkeit nicht fehlen darf und dort erst so richtig zu Hause ist.

Die Idee, aus dem Umgang der Ägypter mit ihrem Schriftsystem ein allgemeineres Prinzip abzuleiten und es „Etymographie“ zu nennen, kam mir, nicht ganz zufällig, in Tokio. Ich hatte dort bei den Germanisten der Keio Universität einen Vortrag über Schillers Ballade „Das verschleierte Bild zu Sais“ und den Begriff des Erhabenen gehalten. In der Diskussion ging dann mein Gastgeber, der Germanist Soichiro Itoda, an die Tafel und schrieb zwei chinesische Schriftzeichen, sogenannte kanji, an, von denen das sehr viel ökonomischere japanische Schriftsystem immerhin 1-2000 für besondere Zwecke, d. h. besondere Begriffe integriert hat. Die beiden Schriftzeichen beziehen sich auf zwei Silben und werden suh-koh ausgesprochen. So würden wir den Sachverhalt darstellen. Nicht aber Prof. Itoda. Keineswegs beziehen sich die beiden Schriftzeichen einfach auf zwei Silben. Sie setzen sich, wenn ich mich recht erinnere, aus zwölf Bildelementen wie etwa Herrscher, Berg, Altar, Aussichtsturm usw. zusammen, die mit den in ihnen evozierten Vorstellungen den japanischen Begriff des Erhabenen bilden. Anhand der Bildlichkeit dieser Schriftzeichen konnte Prof. Itoda ungefähr eine halbe Stunde lang über den japanischen Begriff des Erhabenen extemporieren. ${ }^{2}$

\footnotetext{
1 Assmann 2003.
}

2 Vgl. Itoda 2003. 
Herrn Itodas Ausführungen erinnerten mich sofort an den vielgescholtenen Horapollon, der das richtige Verständnis der Hieroglyphen um Jahrhunderte verzögert hat. Champollion gelang der Durchbruch zur Entzifferung vor allem dadurch, dass er sich entschlossen von Horapollon freimachte, was vor ihm keiner gewagt hatte. Horapollon hat daher in der Ägyptologie keinen guten Ruf, aber in Japan ging mir auf, wie man ihn zu verstehen hat.

Horapollon schrieb zwei Bücher über Hieroglyphen, die im Jahre 1419 von dem italienischen Reisenden Cristofero Buondelmonti in einem Kloster auf der Insel Andros entdeckt und nach Florenz verbracht wurden. ${ }^{3}$ Er war ein Ägypter, der zu Anfang des 5. Jahrhunderts lebte, als die Kenntnis der Hieroglyphenschrift in Ägypten bereits ausgestorben war. Er gehörte zu einer bedeutenden, hoch gebildeten Familie in Akhmim, die u. a. auch den Dichter Nonnos hervorgebracht hatte. Horapollon gibt sein Werk als Übersetzung aus dem Ägyptischen aus, was gewiss eine Fiktion ist, auch wenn Ägyptisch bzw. Koptisch tatsächlich seine Muttersprache war. Das erste Buch (das zweite ist ein späterer, nichtauthentischer Zusatz) behandelt ungefähr 70 Hieroglyphen, die nach heutigem Wissen großenteils richtig gelesen, aber falsch erklärt werden. So schreibt Horapollon z. B. völlig richtig, dass die Ägypter das Bild des Hasen zeichnen, wenn sie den Begriff „öffnen“ schreiben wollen. Falsch ist aber, dass sie das deshalb tun, weil dieses Tier nie seine Augen schließt. Der wahre Grund ist vielmehr, dass das Wort $w-n$ „öffnen“ im Ägyptischen denselben Lautwert wie das Wort $w-n$ (.) „Hasengöttin“ hat. Ein anderes Beispiel ist das Bild der Ente, von dem Horapollon wiederum richtig angibt, dass es das Wort „Sohn“ schreibt. Falsch ist aber, dass sich diese Bedeutung vom besonderen Familiensinn der Ente herleiten soll. Auch hier gilt wieder, dass das ägyptische Wort für Sohn denselben Konsonantenbestand wie das Wort für Ente (bzw. Gans) hat. Der Basilisk bedeutet „Zeit“, weil diese Schlangenart unsterblich ist, dafür mit ihrem Blick und Atem alle anderen Tiere töten kann. Das Prinzip ist klar: Horapollon sieht vom Lautwert der Schriftzeichen völlig ab, von dem er vermutlich auch keine Ahnung mehr hat. Diesen verloren gegangenen Wissenskomplex ersetzt er durch einen anderen Wissensbestand, der eigentlich im klassisch-ägyptischen Schriftsystem keine Rolle spielte, und zwar jene volkstümliche Zoologie, die den Tieren alle möglichen Eigenschaften und Verhaltensformen unterstellt und die wir aus vielen naturkundlichen Werken der Antike kennen wie etwa Aelian, Plinius und Physiologus: dass der Hase nie die Augen schließt, dass die Ente einen besonderen Familiensinn hat, dass der Pelikan seine Jungen mit Herzblut füttert, dass der Löwe ein königliches Tier ist, dass die Schlange unsterblich ist, weil sie sich immer wieder verjüngt usw. usw. Durch diese moralische Zoologie wird es möglich, abstrakte Begriffe wie „öffnen“, „Familie“, „Fürsorge“, „Herrschaft“, „Unsterblichkeit“ usw. in konkreten Bildern dazustellen bzw. zu ,schreiben“. Für Horapollon muss man in diese Art Wissen eingeweiht sein, um die Hieroglyphen verstehen zu können. Wer die Hieroglyphen beherrscht, kennt die Eigenschaften der Dinge, die sie darstellen: das Wesen der Tiere, das Wirken der Natur, den Zusammenhang der Welt. Phonologie wird durch Physiologie ersetzt, Sprachkenntnis durch Weltwissen.

3 Sbordone 1940; Boas 1993; Regni/Zanco 1996; Thissen 2001. 
Bei seinen Hieroglyphenerklärungen verfährt Horapollon nach dem Schema: wenn sie (scil. die Ägypter) den Begriff (A) darstellen wollen, zeichnen sie den Gegenstand oder die Gegenstände (B), weil (C), wobei (A) den Begriff, (B) das hieroglyphische Zeichen und (C) die Erklärung wiedergibt, also z. B. „wenn sie den Begriff ,öffnen“ (A) schreiben wollen, zeichnen sie einen Hasen (B), weil dieses Tier nie seine Augen schließt“ (C), oder, um ein nichtzoologischen Beispiel zu nehmen, ,wenn die Ägypter den Begriff, Ewigkeit' darstellen wollen, zeichnen sie Sonne und Mond, weil sie ewige Elemente sind“. Die Verbindung besteht also nicht zwischen dem Zeichen und dem Bezeichneten, sondern zwischen dem von dem Zeichen abgebildeten Ding und dem Bezeichneten. Hieroglyphen im Sinne Horapollons sind Zeichen, die in einem doppelten Bezug stehen: in symbolischem Bezug zu einem Begriff und in ikonischem Bezug zu einem Gegenstand:

Sonne und Mond funktionieren kraft der natürlichen Beziehung „C“ gewissermaßen als Metaphern der Ewigkeit im Sinne der unaufhörlich in sich kreisenden Zeit, die der Ägypter Neheh nennt. Das japanische Verfahren, wie es Soichiro Itoda in seinem Diskussionsbeitrag erläuterte, lässt sich in die Formel zusammenfassen: wenn die Japaner den Begriff des „Erhabenen“ ausdrücken wollen, dann zeichnen sie die Gegenstände „B“ wie etwa „Herrscher“, „Berg“, „Altar“, Aussichtsturm“ usw., weil die Verbindung dieser Gegenstände für sie die Vorstellung des Erhabenen auf metaphorische Weise veranschaulicht.

Das etymographische Prinzip ergibt sich nun aus der Umkehrung dieses Verfahrens. Nicht der Begriff soll gegeben sein und die Ausdrucksform gefunden werden, sondern die Ausdrucksform soll gegeben sein und von ihr ausgehend soll der Sinn des Begriffs erschlossen werden. Uns ist dieses Verfahren nur von der sprachlichen Ausdrucksform, der Wortbildung her vertraut. Wenn wir nach der Grundbedeutung eines Begriffs fragen, z. B. des Begriffs, Religion', dann ist es ein übliches Verfahren, der Herkunft dieses Wortes auf den Grund zu gehen. Dieser Fall ist interessant, weil man hier auf zwei lateinische Verben ganz verschiedener Bedeutung verwiesen wird: re-ligere und re-ligare. Das eine hängt mit legere ,sammeln, lesen“ zusammen, und wer mit Cicero den Begriff Religion auf dieses Verb zurückführt, gewinnt daraus die semantischen Elemente „Sammlung, Konzentration, Sorgfalt, Aufmerksamkeit“ usw., die das Wortzeichen religio „motivieren“. Das andere Wort hängt mit ligare „binden“ zusammen und heißt soviel wie „zurückbinden“, „wiederanknüpfen“. Wer mit Laktanz diesem Ursprung den Vorzug gibt, gewinnt daraus die Sinnelemente des Bundes, der über Trennung und Abwendung hinweg wiedergewonnenen und aufrecht erhaltenen Verbindung von Gott und Mensch. Hier motivieren die Elemente Bund, Bindung, Verbindlichkeit die Prägung des Wortzeichens religio. Dieses Verfahren nennen wir „Etymologie“, und das Beispiel „Religion“ macht klar, wie viel es herzugeben vermag und wie entscheidend in manchen Fällen die richtige Etymologie für das Verständnis eines Begriffs sein kann. Kommt es auf die Bindung an, auf Treue, Hingabe, Liebe? Oder kommt es auf die Aufmerksamkeit an, auf Andacht, Sorgfalt, Gedächtnis? Wer so fragt, argumentiert von der Motiviertheit des Zeichens her. Die Form des Signifikanten ist eben nicht arbiträr, sondern sagt in ihrer Oberflächenkonstitution schon viel oder geradezu alles über den Sinn, das Signifikat. Rück-Bindung 
oder Rück-Sammlung: das ist ein wichtiger semantischer Unterschied, der sich aus der Etymologie ergibt. Heidegger hat dieses Verfahren bekanntlich philosophisch nobilitiert. Im „Hören auf die Sprache“ erschließt sich ein ursprüngliches Seinsverständnis, das in ihrem alltäglichen Gebrauch nur noch im Modus der Vergessenheit oder Unbewusstheit mitschwingt. So bestimmt Heidegger z. B. von der Etymologie des griechischen Wortes aleitheia her den Begriff „Wahrheit“ unter Verweis auf seine Komponenten „alpha privativum“ und „lethe: Vergessen, Verborgenheit" als „Unverborgenheit“.

Statt auf die Sprache zu hören, blicken die Ägypter bzw. blickt Horapollon auf die Schrift, und da es sich um eine Bilderschrift handelt, blicken sie durch die Schrift hindurch auf die Welt der Dinge und Lebewesen. Dabei sieht Horapollon vom Lautwert der Schriftzeichen völlig ab und behandelt sie rein als Bilder. Darin irrt er, denn die Hieroglyphen funktionieren meist nur über ihren Lautwert. In einem wichtigen Punkt aber hat Horapollon völlig recht: das ist der Punkt, in dem sich seine Idee einer schriftbezogenen Begriffsbildung mit dem japanischen Verfahren einer schriftbezogenen Bedeutungsfindung deckt. Dieses Verfahren, das zugleich eine Denkform ist, nenne ich „Etymographie“. Dieses Verfahren - oder diese Denkform - geht nicht von der Wortgestalt, sondern von der Schreibung aus. Wenn wir uns mit Heideggers Beispiel, dem Begriff „Wahrheit“, an die Ägypter anstatt an die Griechen wenden, dann stoßen wir auf das Wort ma'at, das sowohl „Wahrheit", wie auch „Gerechtigkeit" und „Ordnung" bedeutet und mit dem Zeichen der Feder geschrieben bzw. determiniert wird. Die Etymographie dieses Zeichens führt auf einen Begriff wie „Lufthaftigkeit“, den es im Ägyptischen natürlich als Wort ebenso wenig wie im Deutschen gibt. Wahrheit ist etwas so Unsichtbares, so Allgegenwärtiges aber auch etwas zum Leben so unabdingbar Notwendiges wie die Luft. Wahrheit ist ein alldurchdringendes Lebenselement. So wie Fische im Wasser, leben Menschen in der Luft der Wahrheit. Auch hier müssen wir uns wieder erinnern, dass das ägyptische Wort Ma'at Wahrheit und Gerechtigkeit heißt. Wo Wahrheit-Gerechtigkeit herrscht, herrscht eine „Atmosphäre“, in der Menschen leben können. Die Ägypter bilden diesen, wie ich meine, schönen und eindrucksvollen Begriff der „Lufthaftigkeit“, nicht indem sie ein Kunstwort erfinden, wie ich das im Deutschen versuche, sondern indem sie die Metapher der Feder als Schriftzeichen erfinden. Die Begriffsbildung kann also hier die Sprache ausklammern und direkt sich der Schrift bedienen. Tatsächlich wird in ägyptischen Texten sehr oft die Ma'at mit der Luft verglichen, worauf ich hier nicht eingehen kann. ${ }^{4}$ Die Lufthaftigkeit der Ma'at war in Ägypten eine lebendige Metapher, die Etymographie des Schriftzeichens war den Ägyptern immer bewusst. So legt sich der Verdacht nahe, dass an der etymographischen Methode, wie Horapollon sie praktiziert, vielleicht doch etwas daran sein könnte, auch in Bezug auf die älteren Perioden der ägyptischen Schriftgeschichte. Wo westliche Denker zum Mittel der Sprache greifen, um neue Begriffe zu bilden, da greifen die Ägypter zum Bild.

Die ägyptische Hieroglyphenschrift, das muss nun freilich zunächst einmal gegen Horapollon und alle, die ihm in seiner Deutung der Hieroglyphen gefolgt sind, in aller Deutlichkeit gesagt werden, bezieht sich auf Sprache, und zwar die altägyptische

4 Vgl. die Einzelheiten bei Assmann 2003, $41 \mathrm{f}$. 
Sprache und bleibt ohne die Kenntnis dieser Sprache vollkommen unverständlich. Sie bezieht sich aber nicht, wie die Alphabetschriften, nur auf die lautliche Ebene der Artikulation, sondern darüber hinaus auch noch auf die Sinnebene. Es gibt sogar eine Menge von Zeichen, die gar keinen Laut-, sondern nur einen Sinnwert haben. Das sind die „Determinative“. Hier bedeutet das Bild nicht den dargestellten Gegenstand selbst, sondern eine durch ihn vertretene Sinnklasse. So bezeichnet z. B. ein gehendes Beinpaar die Verben und Begriffe der Bewegung, ein Auge die Begriffe des Sehens, das Bild der Sonne die Zeit und das eines Hausgrundrisses die Raumbegriffe. Die Besonderheit der Determinative oder klassifikatorischen Zeichen liegt in der Abstraktheit ihrer Denotate. In den meisten Fällen bezeichnen sie Begriffe, für die es im Lexikon kein Wort gibt (wie z. B. der Begriff „Lufthaftigkeit“ oder auch Begriffe wie „Zeit“, „Raum“, „Bewegung“, „Säugetier“ (bzw. Vierfüßler) usw.) Das Bild des Krokodils z. B. determiniert eine Klasse von Wörtern wie Bosheit, Aggressivität, Angriff, Raubgier. Damit ist eine Eigenschaft bezeichnet, die im Verhalten des Krokodils exemplarisch hervortritt und die man vielleicht als „Krokodilizität“ umschreiben kann. Der entsprechende Begriff ist im ägyptischen also nur graphisch, aber nicht lexikalisch realisiert. Dieses Zeichen bezieht sich nicht auf ein Wort, sondern auf einen begrifflichen Komplex, der nur in einem mehr oder weniger umfangreichen Diskurs über die Bosheit und Aggressivität zu entfalten wäre. Diese Möglichkeit der Hieroglyphenschrift, mit dem Bild bestimmter Dinge Begriffe beliebiger Komplexität ausdrücken zu können, hat die Griechen so stark beeindruckt, dass sie darin das Wesen der Hieroglyphenschrift überhaupt erblickten.

Das Gegenstück zur Etymographie ist die Ikonographie. So wie die Etymographie auf den Bildsinn der Schrift, bezieht sich die Ikonographie auf den Schriftsinn des Bildes. Die Etymographie nimmt das Schriftbild als Darstellung, die Ikonographie nimmt das Bild als Schreibung eines Sinnkomplexes. Das ist natürlich ein ganz spezieller Gebrauch, den ich hier vom Begriff der Ikonographie mache; er scheint mir aber interessant und für das Projekt Schriftbildlichkeit oder Bildschriftlichkeit einschlägig genug, um zum Schluss wenigstens ganz kurz darauf einzugehen. Auch dieser Begriff ergibt sich aus einer griechischen Deutung oder, wenn man will, Fehldeutung der Hieroglyphenschrift. Die ausführlichste Darstellung dieses Begriffs von Ikonographie als einer Methode, Bilder wie Schrift zu lesen, findet sich bei Plotin:

„Die ägyptischen Weisen [...] verwendeten zur Darlegung ihrer Weisheit nicht die Buchstabenschrift, welche die Wörter und Prämissen nacheinander durchläuft und auch nicht die Laute und das Aussprechen der Sätze nachahmt, vielmehr bedienten sie sich der Bilderschrift, sie gruben in ihren Tempeln Bilder ein, deren jedes für ein bestimmtes Ding das Zeichen ist: und damit, meine ich, haben sie sichtbar gemacht, daß es dort oben [bei den Göttern] kein diskursives Erfassen gibt, daß vielmehr jenes Bild dort oben Weisheit und Wissenschaft ist und zugleich deren Voraussetzung, daß es in einem einzigen Akt verstanden wird und nicht diskursives Denken und Planen ist."

5 Plotin 1964, 49-51; vgl. Stolzenberg 2003, 87f. 
So ist die Stelle zwar kaum verständlich, aber wir verdanken Marsilio Ficino einen Kommentar, der sofort klar macht, worum es hier geht. Er veranschaulichte das von Plotin gemeinte Prinzip der ägyptischen Hieroglyphenschrift am Begriff der Zeit, den die Ägypter im Bilde einer sich in den Schwanz beißenden Schlange dargestellt haben sollen:

„Ihr habt ein diskursives Wissen über die Zeit, das vielfältig und flexibel ist, indem ihr z. B. sagt, daß die Zeit vergeht und nach einem bestimmten Umlauf das Ende wieder an den Anfang knüpft ... Die Ägypter aber fassen einen ganzen Diskurs dieser Art in das einzige Bild einer geflügelten Schlange, die sich in den Schwanz beißt." “6

Der Schriftsinn des Bildes lässt sich nach Plotin als der Diskurs definieren, den das Bild in gedrängter Form symbolisiert und den der Wissende dem Bild ablesen kann. Es handelt sich also um das, was Niklas Luhmann Kompaktkommunikation nennt. ${ }^{7}$ Die Ägypter kannten tatsächlich das Symbol des Uroboros, der sich in den Schwanz beißenden Schlange, allerdings ohne Flügel. Flügel bekommt sie erst im Zusammenhang der Alchemie. Der Uroboros ist aber keine Hieroglyphe und hat nichts mit Schrift zu tun. Er ist ein Bildmotiv und kein Schriftzeichen. Die Ägypter waren in der Tat groß in der Erfindung komplexer symbolischer Bildmotive, die sich in umfangreiche Diskurse übersetzen ließen.

Als den Höhepunkt ikonischer Kompaktkommunikation müsste man aber wohl die romanische Kunst bezeichnen, die es unternahm, hochkomplexe theologische Zusammenhänge wie etwa die Beziehung zwischen dem Alten und dem Neuen Testament in Bildern auszudrücken, die tatsächlich, genauso wie sich Plotin das von der Hieroglyphenschrift vorstellte, gelesen und in Diskurs rückübersetzt werden wollen. Ich möchte das an zwei Beispielen verdeutlichen. Das erste Beispiel stammt aus Vézelay, aus dem 12. Jahrhundert und veranschaulicht die Beziehung zwischen dem Alten und dem Neuen Testament im Bild der „mystischen Mühle“. Oben schüttet Moses die Körner des Gesetzes hinein, unten fängt Paulus das Mehl des Glaubens auf. Das Neue Textament wird so als eine sublimierte, verfeinerte, verarbeitete Form des Alten verstanden. Dieser Gedanke wird hier in die Form eines gedrängten Bildes gebracht, das sich wiederum in umfangreiche Diskurse übersetzen lässt. Das zweite Beispiel stammt aus Chartres, aus dem 13. Jh. von einem der berühmten Glasfenster und stellt die vier Evangelisten dar, die Huckepack auf den Schultern von vier alttestamentlichen Propheten sitzen: Lukas auf Jeremias, Matthäus auf Jesaja, Johannes auf Hesekiel und Markus auf Daniel. Die Propheten haben den Messias vorhergesagt, die Evangelisten und ihre Zeitgenossen haben ihn gesehen, aber nur, weil sie auf den Schultern der Propheten standen, ihre Schriften kannten und dadurch die Zeichen ihrer Zeit zu lesen verstanden. Mit dem neuplatonischen Prinzip des gedrängten Bildes, der fast im Sinne einer Datenkompression zu verstehenden bildlichen Veranschaulichung hochkomplexer theologischer und philosophischer Konzeptionen, entwickelt sich in der christlichen Kunst neben ihrer schon aus

6 Marsilio Ficino, zit. nach Dieckmann 1970, 37.

7 Luhmann 1986, 627; siehe auch Berger 2004. 
der Antike sich herleitenden narrativen Funktion, der Veranschaulichung der biblischen Geschichte, eine lehrhaft-argumentative Funktion, die genau das anstrebt, was Plotin als Leistung der ägyptischen Hieroglyphen beschreibt.

\section{Literatur}

Assmann, Jan (2003): „Etymographie: Zeichen im Jenseits der Sprache“, in: Aleida Assmann und Jan Assmann (Hrsg.), Hieroglyphen. Stationen einer anderen abendländischen Grammatologie, München, S. 37-64.

Berger, Ingrid (2004): „Literatur als Kompaktkommunikation“, in: dies., Musil mit Luhmann. Kontingenz-Roman-System, München, S. 71-91.

Boas, G. (1993): The Hieroglyphics of Horapollo, Bollingen Series XXIII, Princeton.

Dieckmann, Liselotte (1970): Hieroglyphics. The History of a Literary Symbol, St. Louis.

Itoda, Soichiro (2003): „Sinojapanische Schrift als archäologischer Gedächtnisspeicher. Rekonstruktion von Ritualen und Anschauungsräumen am Beispiel des Erhabenen“, in: Aleida Assmann und Jan Assmann (Hrsg.), Hieroglyphen. Stationen einer anderen abendländischen Grammatologie, München, S. 77-88.

Luhmann, Niklas (1986): „Das Kunstwerk und die Selbstreproduktion der Kunst“, in: H. U. Gumbrecht und K. L. Pfeiffer (Hrsg.), Stil, Frankfurt a. M., S. 620-672.

Plotin (1964): Von der geistigen Schönheit (Enneades V 8,6), in: ders., Plotins Schriften, übers. von R. Harder, Bd. 3, Hamburg.

Regni, M. A. und E. Zanco (1996): Orapollo, I Geroglifici, Introduzione, traduzione e note di Mario Andrea Regni e Elena Zanco, Mailand.

Sbordone, Francesco (Hrsg.) (1940): Horapollinis Nilotici Hieroglyphica, 2 Bde., Neapel.

Stolzenberg, Daniel (2003): „Lectio Idealis: Theory and Practice in Athanasius Kircher's Translations of the Hieroglyphs", in: L. Morra und C. Bozzanella (Hrsg.), Philosophers and Hieroglyphs, Turin, S. 74-97.

Thissen, H.-J. (2001): Des Niloten Horapollon Hieroglyphenbuch, Bd. I, Leipzig (u. a.). 